\title{
Supporting Information for \\ Inverse design of metasurfaces based on coupled-mode theory and adjoint optimization
}

Ming Zhou ${ }^{1 *}$, Dianjing Liu ${ }^{1}$, Samuel W. Belling ${ }^{1}$, Haotian Cheng ${ }^{2}$, Mikhail A. Kats ${ }^{1}$, Shanhui $\mathrm{Fan}^{3}$, Michelle L. Povinelli ${ }^{4}$, Zongfu $\mathrm{Yu}^{1}$

1. Department of Electrical and Computer Engineering, University of Wisconsin - Madison, WI 53706, USA

2. Department of Physics, Peking University, Beijing 100871, China

3. Department of Electrical Engineering, Stanford University, Stanford, CA 94305, USA

4. Ming Hsieh Department of Electrical and Computer Engineering, University of Southern California, Los Angeles, CA 90089, USA

* Correspondence to: Ming Zhou ( $\underline{\text { mzhou34@wisc.edu) }}$ 


\section{Inverse design of metasurfaces on substrates}

Here we describe the coupled-mode formalism for metasurfaces on substrates and design a metalens using the developed formalism.

In the presence of substrates, the downward-propagating waves radiated by the metasurface now are partially reflected by the substrate, which in turn changes the resonances amplitudes of the metasurface. As a result, the coupled-mode equations for a metasurface on a substrate then can be written as

$$
\begin{gathered}
\frac{d}{d t} \boldsymbol{a}=\left(j \boldsymbol{\Omega}-\frac{\mathbf{D}^{\dagger} \mathbf{D}}{2}\right) \boldsymbol{a}+\mathbf{D}^{\mathbf{T}}\left(\boldsymbol{S}_{\text {in }}+\mathbf{R}_{\text {sub }} \boldsymbol{S}_{\text {out }}\right) \\
\boldsymbol{S}_{\text {out }}=\mathbf{C}\left(\boldsymbol{S}_{\text {in }}+\mathbf{R}_{\text {sub }} \boldsymbol{S}_{\text {out }}\right)+\mathbf{D} \boldsymbol{a}
\end{gathered}
$$

Here we assume all channels have the same polarization for compactness. $\mathbf{R}_{\boldsymbol{s u b}}$ is a block matrix that can be written as

$$
\mathbf{R}_{\text {sub }}=\left[\begin{array}{cc}
\mathbf{0} & \mathbf{0} \\
\mathbf{0} & \mathbf{r}_{s u b}
\end{array}\right]
$$

where $\mathbf{0}$ is a $\frac{N}{2}$ by $\frac{N}{2}$ matrix with its all elements being zero, and $N$ is the number of channels. $\boldsymbol{r}_{\boldsymbol{s u b}}$ is a $\frac{N}{2}$ by $\frac{N}{2}$ anti-diagonal matrix with its anti-diagonal elements being the reflection coefficient of the substrate.

In steady state, we substitute $\boldsymbol{a}=\boldsymbol{a}\left(\omega_{0}\right) e^{j \omega_{0} t}$ and $\boldsymbol{S}_{\boldsymbol{i n}}=\boldsymbol{S}_{\boldsymbol{i n}}\left(\omega_{0}\right) e^{j \omega_{0} t}$ into Eq. S1-2, and then obtain:

$$
\boldsymbol{S}_{\text {out }}\left(\omega_{0}\right)=\left[\mathbf{C}+\mathbf{D}\left(j \omega_{0} \mathbf{I}_{M \times M}-j \boldsymbol{\Omega}+\frac{\mathbf{D}^{\dagger} \mathbf{D}}{2}\right)^{-\mathbf{1}} \mathbf{D}^{\mathbf{T}}\right]\left(\boldsymbol{S}_{\text {in }}\left(\omega_{0}\right)+\mathbf{R}_{\text {sub }} \boldsymbol{S}_{\text {out }}\left(\omega_{0}\right)\right)
$$

where $\mathbf{I}_{M \times M}$ is $M$ by $M$ identity matrix. Then the amplitudes of the outgoing plane wave $\boldsymbol{S}_{\text {out }}\left(\omega_{0}\right)$ from the metasurface can be obtained as

$$
S_{\text {out }}\left(\omega_{0}\right)=\left[\mathbf{I}_{N \times N}-\mathbb{S} \mathbf{R}_{\text {sub }}\right]^{-\mathbf{1}} \mathbb{S} \boldsymbol{S}_{\text {in }}\left(\omega_{0}\right)
$$

where $\mathbf{I}_{N \times N}$ is $N$ by $N$ identity matrix. Here we define $\mathbb{S}=\mathbf{C}+\mathbf{D}\left(j \omega_{0} \mathbf{I}_{M \times M}-j \boldsymbol{\Omega}+\frac{\mathbf{D}^{\dagger} \mathbf{D}}{2}\right)^{-\mathbf{1}} \mathbf{D}^{\mathbf{T}}$ for compactness.

The amplitudes of the waves transmitted through the substrate $\boldsymbol{S}_{\text {trans }}\left(\omega_{0}\right)$ then can be obtained as $\boldsymbol{S}_{\text {trans }}\left(\omega_{0}\right)=\mathbf{t}_{\text {sub }} \boldsymbol{S}_{\text {out }}^{\text {down }}\left(\omega_{0}\right)$, where $\mathbf{t}_{\text {sub }}$ is a $\frac{N}{2}$ by $\frac{N}{2}$ anti-diagonal matrix with its anti-diagonal elements being the transmission coefficient of the substrate and $\boldsymbol{S}_{\text {out }}^{\text {down }}\left(\omega_{0}\right)$ is the amplitudes of downward-propagating waves from the metasurface. Once we obtain the transmitted waves $\boldsymbol{S}_{\text {trans }}\left(\omega_{0}\right)$, the field distribution after the substrate can be calculated and designed following the same adjoin-optimization approach described in the main text. 
As a specific example, we designed a metalens on a substrate. As shown in Fig. S1a, the thickness of the substrate is $3.5 \lambda_{0}$, where $\lambda_{0}$ is the working wavelength. The refractive index of the substrate is 1.5. The metalens has a size of $200 \lambda_{0}$ and consists of 401 different infinitely long square nanorods placed in a line. For simplicity, the refractive index of the nanorods is fixed to be 4 . Similar to the metalens described in Fig. 3, the parameters to be designed here include the side length $l_{m}$ and the location $x_{m}$ of each nanorod. The designed focal point is $\left|y_{F}\right| \cong 49 \lambda_{0}$ from the substrate.

(a)

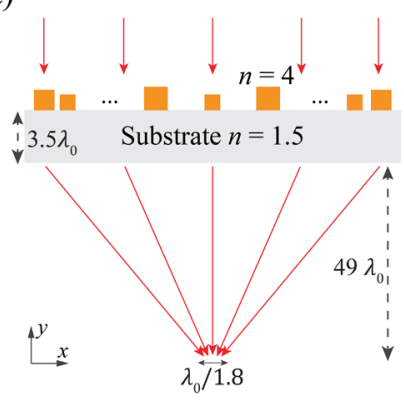

(b)

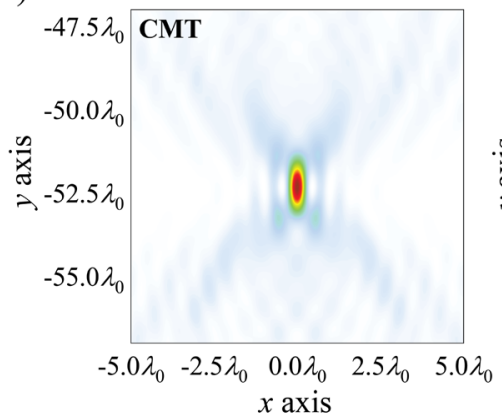

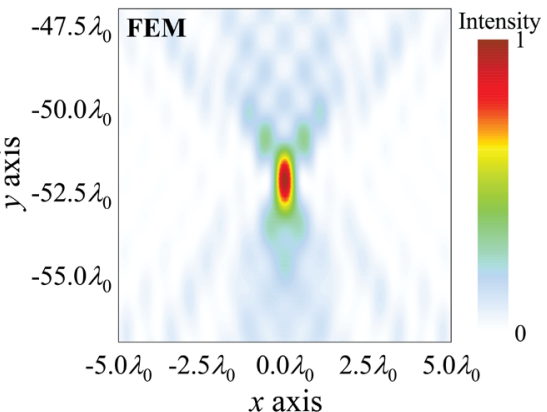

Fig. S1 (a) Schematic of a metalens on a substrate. The metasurface consists of 401 square nanorods with a refractive index of 4 . Here we consider the fundamental TE modes. The substrate has a refractive index of 4 and a thickness of $3.5 \lambda_{0}$, where $\lambda_{0}$ is the working wavelength. (b) Comparison of the field intensities calculated using CMT (left) and FEM (right). The results agree well with each other.

Figure S1b show the field intensities around the designed focal point for the designed metalens on the substrate. The result obtained from the modified CMT (Fig.S1c) agrees well with that from FEM simulation, validating the modified coupled-mode formalism.

\section{Performance of the CMT approach under different configurations}

Here we compare the performance of our approach under different configurations.

Figure S2 compares the performance of our approach for resonances with different radiation profiles and different quality factors. For consistency, we consider the fundamental TE mode and first-order TM mode supported by square nanorods with different refractive indices. The fundamental TE mode has an isotropic radiation profile (Fig. S2a), and the first-order TM mode has a dipolar radiation profile (Fig. S2b and c). For refractive index of 4, the quality factors of the TE and TM mode are 6.87 and 6.85 , respectively. For refractive index of 2.5 , the quality factor of the TM mode is 3.2. We designed 3 metalenses based on these resonance modes and plot the field intensity profiles in Fig. S2d-f. Despite the differences in radiation profiles and quality factors, our approach always shows excellent agreement with FEM simulations. 
(a)

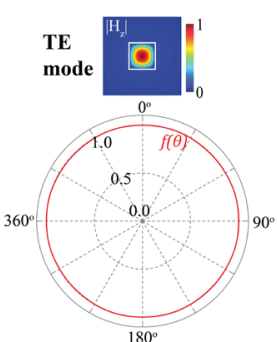

(b)

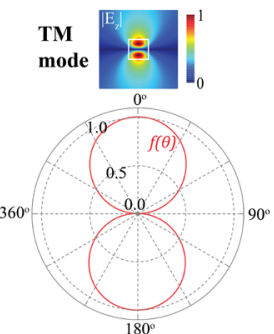

(c)

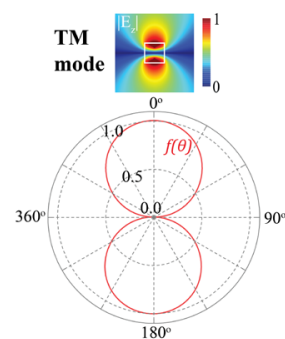

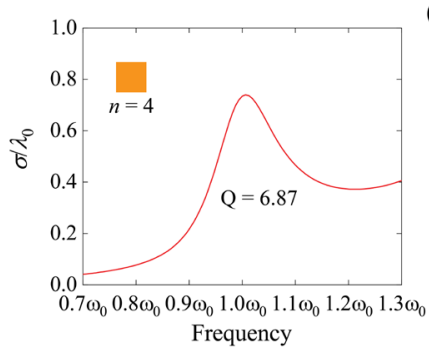

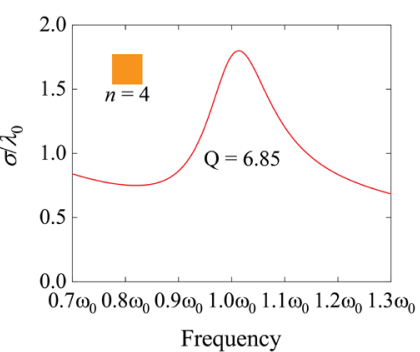

Frequency

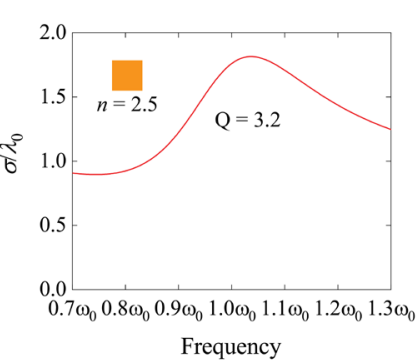

(d)
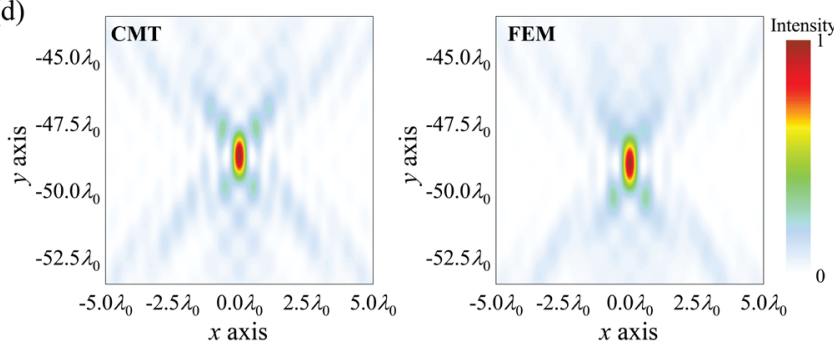

(e)
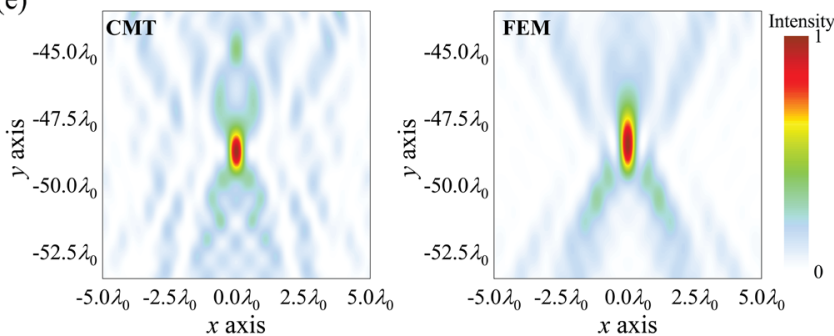

(f)

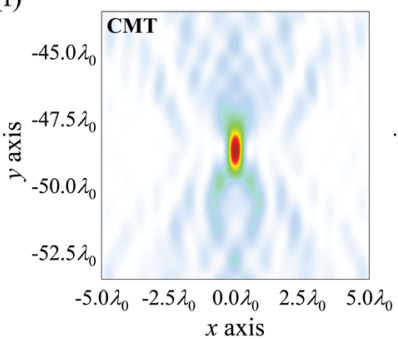

Fig. S2 (a-c) Modal profiles, radiation profiles, and spectra for different resonances. (d-f) Intensity profiles of metalens designed using the resonances in (a-c). The results from CMT and FEM simulations agrees well with each other.

Figure S3 compares the performance of our approach for metalens with the same size, but different number of elements. The size of the metalens is fixed at $200 \lambda_{0}$ and we consider the fundamental TE modes supported by square nanorods. For the metalens with 501 nanorods, the spacing between adjacent nanorods is $0.4 \lambda_{0}$. For the metalens with 1001 nanorods, the spacing between adjacent nanorods is $0.2 \lambda_{0}$. The couplings between nanorods under such spacings are quite different. However, for both cases, our CMT approach agrees well with the FEM simulation. 


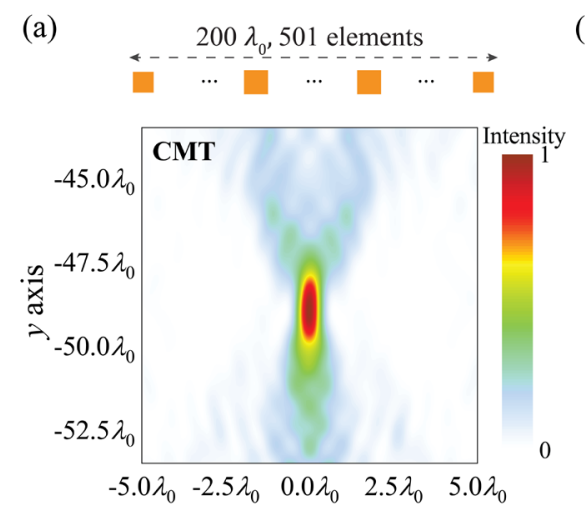

(b) $\quad 200 \lambda_{0,}, 1001$ elements $\ldots$
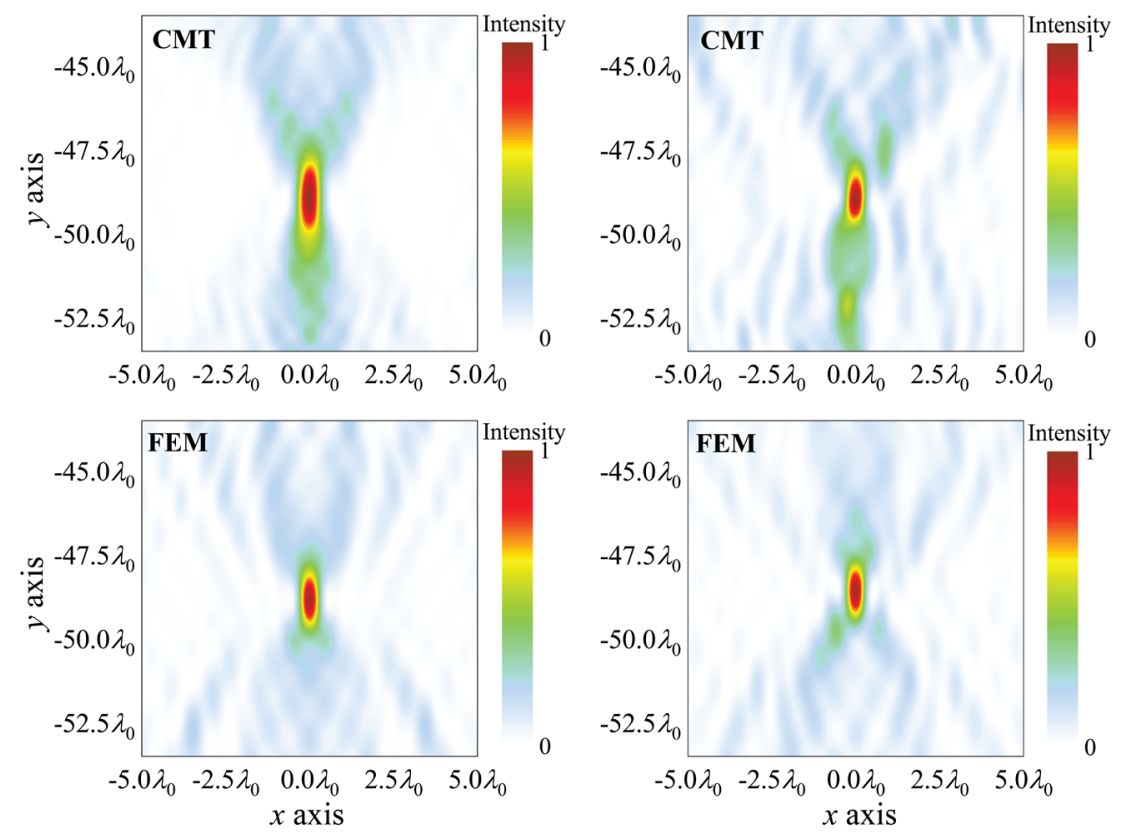

Fig. S3 Comparison between CMT and FEM for metalenses with the same size but different number of elements. For both cases, CMT agree well with FEM simulations.

Table S1 further summarizes the focusing efficiencies under the above 5 different configurations. For all configurations, the focusing efficiency predicted by CMT agree well with that from FEM simulations.

Table S1. Focusing efficiencies predicted by CMT and calculated by FEM

\begin{tabular}{cccccc}
\hline $\begin{array}{c}\text { Resonance } \\
\text { mode }\end{array}$ & & $\begin{array}{c}\text { TE } \\
(\mathbf{Q}=\mathbf{6 . 8 7})\end{array}$ & & $\begin{array}{c}\text { TM } \\
(\mathbf{Q}=\mathbf{6 . 8 2})\end{array}$ & $\begin{array}{c}\text { TM } \\
(\mathbf{Q}=\mathbf{3 . 2})\end{array}$ \\
\hline $\begin{array}{c}\text { Number of } \\
\text { elements }\end{array}$ & 401 & 501 & 1001 & 401 & 251 \\
CMT & $13.2 \%$ & $8.6 \%$ & $9.8 \%$ & $8.9 \%$ & $7.1 \%$ \\
FEM & $14.7 \%$ & $7.2 \%$ & $8.8 \%$ & $9 \%$ & $6 \%$ \\
\hline
\end{tabular}

